\title{
Assessment of the bio-preparedness and of the training of the French hospital laboratories in the event of biological threat
}

A Mérens ${ }^{1}$, J D Cavallo², F Thibault³, F Salicis4, J F Munoz ${ }^{5}$, R Courcol (rene.courcol@chru-lille.fr) ${ }^{6}$, P Binder ${ }^{7}$

1. Hôpital Bégin, Service de Santé des Armées (Military Health Service), Saint-Mandé, France

2. Ecole du Val-de-Grâce, Service de Santé des Armées (Military Health Service), Paris, France

3. Institut de Recherche Biomédicale des Armées (French Armed Biomedical Research Institute), Grenoble, France

4. Direction Générale de la Santé (General Direction of Health), Paris, France

5. Laboratory of Hydrology of Nancy, Agence Nationale de Sécurité Sanitaire de l'Alimentation, de l'Environnement et du Travail (ANSES, French Agency for Food, Environmental and Occupational Health and Safety), Maisons-Alfort Cedex, France

6. Institute of Microbiology, Centre Hospitalier Régional Universitaire de Lille,(Lille University Medical Center), University of Lille Nord de France, Lille, France

7. Institut National de la Santé et de la Recherche Médicale (INSERM, National Institute of Health and Medical Research), Paris, France

A national laboratory network 'Biotox-Piratox' was created in 2003 in France with the purpose of detecting, confirming and reporting potential biological and chemical threat agents. This network is divided into three levels: Level 1 is dedicated to the evaluation of risks (biological, chemical, radiological), to sampling and packing. Level 2 consists of university and military hospitals, who deal with biological specimens, and of environmental and veterinary laboratories, who deal with environmental and animal samples. Level 3 comprises national reference laboratories and the Jean Mérieux biosafety level (BSL)-4 laboratory in Lyon. This report presents the results of four biopreparedness exercises to check critical points in the processing of samples. These exercises took place in 2007, 2009, 2010 and 2011. Each of them consisted of two parts. The first part was the identification of an unknown bacterial strain and its susceptibility to antibiotics used as a default in case of a bioterrorist event. The second part was the detection of Class III microorganisms, mainly by molecular techniques. The main lesson learnt in these exercises was that the key to successful detection of biological agents in case of a biological threat was standardisation and validation of the methods implemented by all the laboratories belonging to the network.

\section{Introduction}

A national laboratory network 'Biotox-Piratox' was created in France in 2003 with the purpose of detecting, confirming and reporting potential biological and chemical threat agents $[1,2]$. This network is an interministerial laboratory response network organised in three levels (Figure), similar to the surveillance laboratory network in the United States (US) [3-6]. A scientific advisory committee of the Biotox-Piratox network was appointed and entrusted with the three following missions: coordination of the network, training of the laboratories, and monitoring diagnostic tests in development. Since 2007 , the committee has organised four inter-laboratory exercises to check critical points in the processing of samples: transport and reception, traceability, the diagnostic tests implemented, the reactivity and involvement of laboratories. The aim of this report is to present the results of the four biological exercises and the lessons learnt.

\section{Methods}

The exercises, which took place in 2007, 2009, 2010 and 2011, were essentially directed at the second level laboratories. The number of participating labs in these years varied from 27 to 28 . Sixteen laboratories used the same diagnostic equipment and techniques: nine university hospitals, six military hospitals and one military research centre (Institut de Recherche Biomédicale des Armées, IRBA). The other participating centres dedicated to processing environmental samples used equipment and techniques which were more heterogeneous.

The authorisations for transporting and holding samples were obtained from the French national agency for the security of medicines and health products (Agence nationale de sécurité du médicament et des produits de santé; ANSM) for all exercises. All samples were dispatched to the participating centres using a specialised carrier according the French and European regulations. The second exercise was conducted in partnership with the Robert Koch Institute (RKI, Berlin, Germany), and the authorisations were obtained according to the German and French regulations for the transport of the biological material from Berlin (Germany) to Paris (France). For each sample, a scenario describing a realistic situation 
The national Biotox-Piratox laboratory network in France

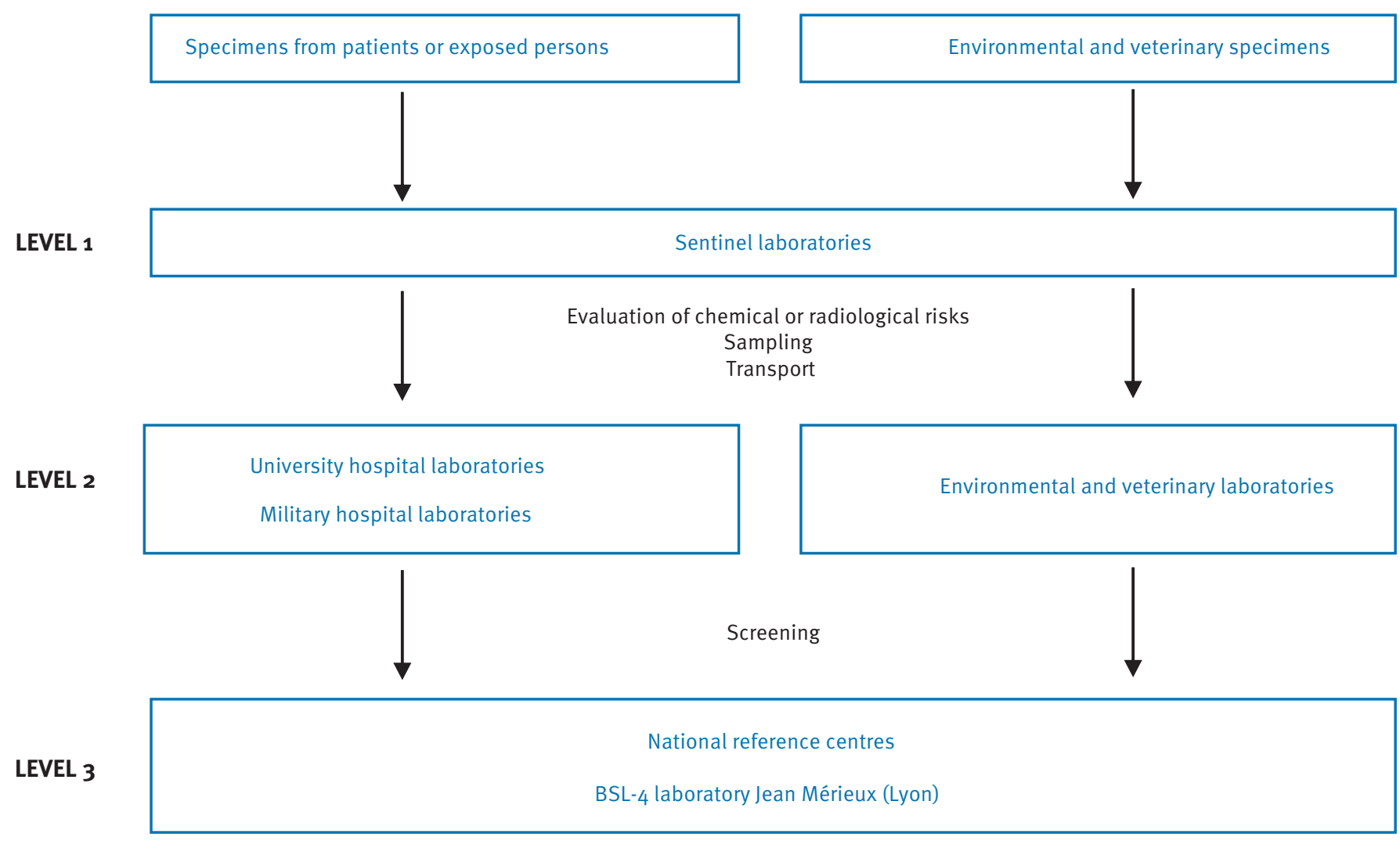

Confirmation and expertise for positive samples

and the conditions of sampling were provided to the laboratories to guide their analytic strategy. All results were collected in a coordinating centre and were analysed by members of the Scientific Advisory Committee of the Biotox-Piratox laboratory network.

\section{First exercise (2007)}

The first exercise focused on the transport of specimens, the turnaround time for detection as well as the specificity and the sensitivity of the techniques implemented. The biological agents that were to be detected were a Bacillus thuringiensis strain resistant to ciprofloxacin (identification and susceptibility testing) and B. anthracis DNA cloned into an Escherichia coli strain (detection by PCR) [7].

\section{Second exercise (2009)}

The main objectives of the second exercise were to create relationships with other European countries, here with the Robert Koch Institute (Germany), to cope with administrative and logistic problems (transport or import), to list the various diagnostic techniques available, to evaluate the performance of diagnostic methods and to motivate participating centres to continue the training of technicians and biologists. The laboratories had to detect different concentrations of the following agents: Francisella tularensis subsp. holartica, irradiation-inactivated monkeypox virus (Mpx) and live vaccinia virus (Vac). The bacterial and viral samples were prepared and quantified by the Centre for Biological Safety at the RKI. It was confirmed that $F$. tularensis samples were viable for at least two weeks if stored at $+4{ }^{\circ} \mathrm{C}$ in phosphate-buffered saline (PBS). The stability of the viral samples was controlled after four weeks at $+4^{\circ} \mathrm{C}$. The samples were transported by plane from Berlin to Paris in a single parcel, and then picked up by the French gendarmerie and taken to a reference laboratory, where the samples were divided into aliquots and dispatched by the police force to all participating centres.

\section{Third exercise (2010)}

The goal of the third exercise was to evaluate the performance of DNA-based techniques and the involvement of a national reference centre at the expertise level (Level 3) for positive samples detected at Level 2. This exercise consisted of a strain of Yersinia pseudotuberculosis (identification and susceptibility testing) and a DNA extract of $Y$. pestis EV76 (detection by $P C R$ ) [8]. At the end of the exercise, the DNA extracts were sent back to the IRBA laboratory to evaluate the performance of the DNA extraction method used by the participating laboratories. Ct (cycle threshold) values were obtained from real-time PCR amplifications 
performed three times. The caf1 gene was the criterion for this evaluation. In addition, positive samples were sent for confirmation to the Plague National Reference Laboratory (Dr. E. Carniel, Institut Pasteur, Paris).

\section{Fourth exercise (2011)}

At the annual seminar in 2011 , it was decided to make the fourth exercise harder. Two Class III microorganisms were mixed together in the same medium: an $E$. coli strain modified to carry the plasmid pXO1 (gene pag) of $B$. anthracis, and the vaccine strain $\mathrm{EV}_{7} 6$ of $Y$. pestis modified to pgm-, pYV+, pFra+, pPst+, in which the caf and pla genes had been replaced by the gene conferring resistance to kanamycin and zeocin. The second part of the exercise consisted in the identification and susceptibility testing of a strain of Burkholderia thailandensis.

\section{Results}

Overall, 22, 26, 28 and 27 laboratories took part in the four exercises, respectively. The results were compared with the expected results, defined as a correct analytical result associated with a correct interpretation.

In all four exercises, parcels were transported and delivered without major problems, except for two cases. The first was a zip code unknown to the carrier. The second was the lack of authorisation of the carrier to transport Class III microorganisms to La Réunion. The delivery time was considered as acceptable (less than 24 hours) in metropolitan France.

At the end of every exercise, samples and waste were destroyed by autoclaving at $134{ }^{\circ} \mathrm{C}$ for $18 \mathrm{~min}$, or at $121{ }^{\circ} \mathrm{C}$ for one hour, or by complete immersion in $2.5 \%$ chlorine when an autoclave was not available (e.g. in field laboratories).

\section{First exercise (2007)}

The results of the first exercise are summarised in Table 1. The first sample contained a strain of $B$. thuringiensis (Bacillus of the cereus group genetically close to $B$. anthracis) resistant to ciprofloxacin. Results were submitted from 22 centres; they took between 28 hours and 15 days to complete the exercise. Phenotypic identification was achieved by all 22 laboratories except for two. The identification systems used were API 20 and API 50CHB (bioMérieux, France; $n=18$ laboratories), Vitek-2 (bioMérieux, France; $\mathrm{n}=2$ laboratories) and Phoenix (Becton-Dickinson, US; $n=1$ laboratory; one laboratory used more than one method). B. thuringiensis was correctly identified in all except one laboratory which falsely identified it as $B$. anthracis. Antibiotic susceptibility testing was carried out by 17 laboratories, of which 16 detected a resistance to ciprofloxacin and 14 confirmed a susceptibility to doxycycline.

The second part of the first exercise consisted of $B$. anthracis DNA (strain Sterne) cloned into an $E$. coli strain. $B$. anthracis strain Sterne is peculiar in that it harbours the gene pagA (encoding protective antigen)
TABLE 1

Results of the first exercise assessing the performance of the Biotox-Piratox laboratory network: identification of Bacillus strains, France, 2007

\begin{tabular}{|l|l|}
\hline First sample & $\begin{array}{l}\text { Bacillus thuringiensis } \\
\text { resistant to ciprofloxacin }\end{array}$ \\
\hline Participants & 22 \\
\hline Correct analytical result & 20 \\
\hline False interpretation & $1 \quad$ B. anthracis \\
\hline Interpretation not done & 1 \\
\hline Results for antibiogram & $16 / 17$ \\
\hline Second sample & DNA Bacillus anthracis \\
\hline Participants & 19 \\
\hline Correct analytical results by PCR & 19 \\
\hline
\end{tabular}

but not the gene capA (encoding capsule). This analysis was carried out by 19 laboratories with satisfactory results (Table 1 ). The results of this second part were returned after a period of three hours to 15 days. Two laboratories also looked for the presence of a chromosomal Bacillus gene (ba813). Only six laboratories drew the accurate conclusion that the strain was the vaccine strain Sterne based on its genotypic characteristics.

\section{Second exercise (2009)}

For the second exercise, the results were variable both for bacterial and viral samples (Table 2). For the identification of Francisella tularensis subsp. holartica, several techniques were implemented and associated: culture (16 laboratories), PCR (21 laboratories), sequencing (four laboratories), immunochromatography (eight laboratories). Susceptibility testing for bacteria was performed by 18 of 25 participants.

Of the 21 laboratories participating in the exercise for viral samples, 20 used a genotypic method, two used culture on Vero cells, and three used sequencing or pyrosequencing. No false positive result was obtained for viral samples, but a lack of sensitivity of the realtime PCR was observed for the lowest viral loads (Table 2).

\section{Third exercise (2010)}

For the third exercise, 28 laboratories participated. The samples were delivered in less than 24 hours except for the transport to one laboratory which was overseas (La Réunion). The delays in obtaining the results have varied between 6.5 hours and eight days. Most of the laboratories $(n=20)$ delivered the results in less than three days. The detection of $Y$. pestis in four samples with different DNA concentrations was performed accurately by only 19 of 28 laboratories (Table 3). Two of the three laboratories using $16 \mathrm{~S}$ DNA sequencing gave a wrong identification. To determine the performance of DNA extraction methods, 28 extracts were sent to the IRBA laboratory for detection of the caf1 gene (Table 4). A maximal difference of $12.72 \mathrm{Ct}$ between 
TABLE 2

Results of the second exercise assessing the performance of the Biotox-Piratox laboratory network: determination of bacterial and viral concentrations, France, 2009

\begin{tabular}{|c|c|c|c|c|c|}
\hline Bacteriology & \multicolumn{5}{|c|}{ Francisella tularensis LVS } \\
\hline Dilution & B1 & B2 & B3 & B4 & B5 \\
\hline Cell count $/ \mathrm{mL}$ & $5 \times 10^{6}$ & $5 \times 10^{6}$ & $5 \times 10^{3}$ & 0 & $5 \times 10^{6}$ \\
\hline Participants & 25 & 26 & 26 & 26 & 26 \\
\hline Correct analytical result & 24 & 26 & 25 & 23 & 25 \\
\hline False interpretation & 0 & 0 & 0 & 0 & 0 \\
\hline Virology & \multicolumn{5}{|c|}{ Monkeypox inactivated by irradiation } \\
\hline Dilution & $V_{1}$ & & & $\mathrm{~V}_{4}$ & $V_{5}$ \\
\hline $\mathrm{pfu} / \mathrm{mL}$ & $1,4 \times 10^{4}$ & & & $2,8 \times 10^{7}$ & $4,1 \times 10^{6}$ \\
\hline Participants & 21 & & & 21 & 21 \\
\hline Correct analytical result & 15 & & & 20 & 20 \\
\hline \multirow[t]{2}{*}{ False interpretation } & 2 & & & 4 & 4 \\
\hline & \multicolumn{5}{|c|}{ Vaccinia virus in PBS, stable and infectious } \\
\hline Dilution & $V_{2}$ & & & V6 & $\mathrm{v}_{7}$ \\
\hline $\mathrm{pfu} / \mathrm{mL}$ & 0 & & & $2,3 \times 10^{3}$ & $4 \times 10^{5}$ \\
\hline Participants & 21 & & & 21 & 21 \\
\hline Correct analytical result & 21 & & & 16 & 20 \\
\hline False interpretation & 0 & & & 2 & 4 \\
\hline
\end{tabular}

LVS: live vaccine strain; PBS: phosphate-buffered saline; pfu: plaque forming units.

the laboratories was observed. This highlights the importance of the extraction step including an optional pretreatment step (lysis), the sample volume used for extraction, the extraction method chosen (manual or automated) and its intrinsic performance for yield and purity of nucleic acid isolation, as well as the eluate volume. Detection of the caf1 gene was possible in 26 extracts from samples with high concentrations, but only in 18 extracts from samples with low concentrations. It should be noted that in some cases IRBA detected caf 1 in extracts that had tested negative in the laboratories. These observations highlight the differences in the performance of the amplification step depending on the protocol used (detection threshold), but could also reflect differences in technical training.

\section{TABLE 3}

Results of the third exercise assessing the performance of the Biotox-Piratox laboratory network: detection of Yersinia pestis EV76 DNA, France, 2010

\begin{tabular}{|l|c|c|c|c|c|}
\hline \multirow{2}{*}{ Bacteriology } & \multicolumn{5}{|c|}{ Yersinia pestis EV76 } \\
\cline { 2 - 6 } & A & B & C & D & All \\
\hline Cell count/mL & $10^{2}$ & 0 & $10^{5}$ & $10^{3}$ & - \\
\hline Participants & 28 & 28 & 28 & 28 & 28 \\
\hline Correct analytical results & 26 & 27 & 24 & 22 & 19 \\
\hline
\end{tabular}

The second part of the exercise was the identification of $Y$. pseudotuberculosis. This microorganism was accurately identified by all laboratories. Identification methods were either manual (API strips, bioMérieux, France) or automated (Vitek-2, bioMérieux, France). Two laboratories used mass spectrometry to identify the strain [9]. Ten laboratories confirmed their result by DNA sequencing. However, sequencing of $16 \mathrm{~S}$ rRNA did not allow distinguishing $Y$. pestis from $Y$. pseudotuberculosis. Antibiotic susceptibility testing was performed either with discs, E-test strips (bioMérieux, France) or Vitek-2 cards (bioMérieux, France).

\section{Fourth exercise (2011)}

This exercise consisted of the identification and susceptibility testing of a strain of $B$. thailandensis (Table 5). A small majority of laboratories identified this isolate correctly at the species level. Depending on the biochemical identification method, $B$. thailandensis could easily be misidentified as $B$. pseudomallei. Only the arabinose test can distinguish these two species. Various methods of identification were employed: phenotypic methods alone or in association with either mass spectrometry or PCR and DNA sequencing. Mass spectrometry was used by four laboratories and gave an accurate identification in all four cases.

As expected, the second part of this exercise was more difficult. Only one third of the laboratories gave a correct result (Table 6 ). These nine laboratories identified both the plasmid $\mathrm{pXO} 1$ cloned in a strain of $E$. coli and the modified vaccine strain of $Y$. pestis. The results of 
TABLE 4

Results of the third exercise assessing the performance of the Biotox-Piratox laboratory network: comparison of Yersinia pestis DNA extracts by detection of the caf1 gene, France, 2010

\begin{tabular}{|l|c|c|c|}
\hline Ct values & $10^{5}$ & $10^{3}$ & $10^{2}$ \\
\hline Ct values with the reference procedure (3 tests) & $25.03-25.18$ & $31.62-32.74$ & $35.23-36.49$ \\
\hline Mean Ct values & 25.79 & 33.54 & 34.99 \\
\hline Lowest Ct values & 19.99 & 27.08 & 30.04 \\
\hline Highest Ct values & 30.54 & 39.81 & 41.19 \\
\hline Laboratories with expected result & 26 & 22 & 18 \\
\hline
\end{tabular}

Ct: cycle threshold.

the other laboratories were incomplete or wrong. Once it is grown, the strain of $Y$. pestis can be identified either by a phenotypic method, by a molecular method or by mass spectrometry. Among the phenotypic methods, the rhamnose test allowed to differentiate this modified $Y$. pestis isolate from $Y$. pseudotuberculosis. As in the third exercise, it was not possible to distinguish the two Yersinia species, neither by molecular methods, nor by mass spectrometry or sequencing.

\section{Discussion}

The main challenge of these exercises, as also performed by the Laboratory Response Netwok (LRN) in the US [10], was to assess the capacity and competence of Level 2 laboratories through relevant exercises involving unknown samples within the framework of the national regulation applied to microorganisms of Class III [11]. Several lessons can be learnt concerning the organisation and the implementation of the exercises, and the methods and techniques that should be used by Level 2 laboratories.

For each exercise, getting the authorisations for transportation and delivery to all laboratories was a long process which required continuous liaison between the Ministry of Health, the French regulatory authority (ANSM) and the organisers. Although all participants agreed that the regulations need to be strict, they were of the opinion that the administrative procedures should be simplified for such exercises. As laboratory exercises on biological threat agents are important with a view to biodefense, exercises and proficiency tests should be integrated in the national regulations. Moreover, as no other European country runs such exercises, it would be worth implementing them under the auspices of European governments.

Since 2002, participating university hospitals have used the same molecular methods with the same primers and probes, the same PCR material, equipment and positive controls. Equipment of military hospital laboratories was different from that used in university hospitals, but all military hospitals used the same equipment, the same primers and probes, and followed similar methodologies which made it possible to ensure coherent results, while non-hospital laboratories (mainly those dedicated to environmental and veterinary samples) showed a larger disparity in the methods and, therefore, in the results. However, the general disparity in the extraction methods (type of protocols and commercial extraction kits, manual or automated methods) has to be underlined.

All methods used to identify bacteria were appropriate for identification at genus and species level, but identification of the species is not sufficient to the government or health authorities to make a decision. Antibiotic susceptibility testing results are important to validate the treatment and implement a public health strategy for antimicrobial treatment and prophylaxis management. Two classes of antibiotics have to be tested because they are recommended as first line therapy or prophylaxis in the event of a biological threat: fluoroquinolones and doxycycline. The minimal inhibitory concentration can easily be determined by the E-test method [12-13]. During the exercises, antibiotic susceptibility results were not obtained systematically from the participants. Consequently, the Scientific Advisory Committee of the Biotox-Piratox laboratory network recommends determining minimal inhibitory concentrations for field and environmental laboratories. If this technique is not available in some

\section{TABLE 5}

Results of the fourth exercise assessing the performance of the Biotox-Piratox laboratory network: identification of Burkholderia thailandensis, France, 2011

\begin{tabular}{|c|c|}
\hline First sample & Burkholderia thailandensis \\
\hline Participants & 27 \\
\hline $\begin{array}{l}\text { Correct analytical } \\
\text { result }\end{array}$ & 16 \\
\hline False identifications & $\begin{array}{l}1 \text { Aneurinibacillus aneurinilyticus } \\
1 \text { B. pseudomallei, Pseudomonadaceae } \\
3 \text { B. cepacia } \\
1 \text { Moraxella lacunata } \\
1 \text { Yersinia pestis PCR-positive } \\
1 \text { Y. pestis PCR-negative } \\
3 \text { no identification }\end{array}$ \\
\hline $\begin{array}{l}\text { Antibiogram } \\
\text { performed }\end{array}$ & $10 / 27$ \\
\hline
\end{tabular}




\section{TABLE 6}

Results of the fourth exercise assessing the performance of the Biotox-Piratox laboratory network: detection of Yersinia pestis (vaccine strain modified) and pXO1 in Escherichia coli in a mix of microorganisms, France, 2011

\begin{tabular}{|c|c|}
\hline Second sample & Yersinia pestis + Escherichia coli $\left(\mathrm{pXO}_{1}\right)$ \\
\hline Participants & 27 \\
\hline $\begin{array}{l}\text { Correct analytical } \\
\text { results with } \\
\text { interpretation }\end{array}$ & 9 \\
\hline Other results & $\begin{array}{ll}1 & \text { Y. pestis } \\
1 & \text { Bacillus spp. } \\
2 & \text { Y. pseudotuberculosis } \\
1 & \text { E. coli } \mathrm{O}_{157: \mathrm{H} 7} \\
4 & \text { E. coli } \\
1 & \text { Clostridium botulinum toxin B } \\
1 & \text { staphylococcal enterotoxin not detected } \\
5 & \text { pagA only detected } \\
2 & \text { Y. pestis only detected }\end{array}$ \\
\hline
\end{tabular}

environmental laboratories, attention is drawn on the importance of rapidly sending the isolate to a hospital laboratory.

Molecular methods were widely used for the identification of bacteria and viruses. The third exercise underlined that the limitations of the PCR method were mainly related to the yield and quality of nucleic acid extraction, to contamination during the PCR and to the sensitivity of the method. As expected, the best results were achieved for the samples with the highest nucleic acid concentrations, especially for viral samples. The worst results were associated with the lowest viral loads, especially for the vaccinia virus sample $\left(V_{3}\right)$ containing only traces of vaccine DNA $\left(\leqslant 10^{2} \mathrm{pfu} / \mathrm{mL}\right)$. The DNA extraction step must be optimised to improve sensitivity. Another problem observed for some centres was the lack of interpretation or unsuitable interpretation accompanying the results. Indeed, the results are intended to be used by the government or health authorities, and must be interpreted and clearly explained so that non-specialists can understand them. This is critical to making accurate decisions.

The organisers set some traps, especially in the fourth exercise. The first trap was the combination of two important pathogens in the same sample, which could happen in case of a bioterrorist attack. The second trap was the modified vaccine strain which required primers different from those used in the previous exercises. The last trap was the presence of a plasmid cloned into an $E$. coli strain, which was overlooked by a number of laboratories.

The conclusion that can be drawn from these exercises is that the key to successful detection of biological agents in case of a biological threat is the training of the laboratory personnel (microbiologists and technicians) dealing with class III organisms as well as the standardisation and validation of methods implemented by all laboratories of the network. This last point was exemplified during the third exercise for molecular methods. However, the French Biotox-Piratox laboratory network is a network of networks. Thus it is not possible or realistic to force all laboratories to use identical equipment or techniques. Nevertheless, it is important to provide specific guidelines to all laboratories involved in the network. The publication of such guidelines for biological specimens is in progress. Moreover, administrative procedures should be adapted to accommodate for national exercises.

Acknowledgments

The authors thank Florent Sebbane (Inserm U1019CNRS82-04) for the Yersinia pestis strain used in the fourth exercise.

References

1. Binder P, Brucker G, Josseran L. [From alert to laboratory: a coherent network designed to deal with to naturally occurring infectious disease outbreaks and bioterrorism.] Bull Acad Natl Med. 2007;191(6):1005-18. French.

2. La coordination départementale et zonale. [Departmental and zonal coordination]. Bordeaux: Gestion des risques exceptionnels et NRBC. [Management of exceptional and CBRN risks]. [Accessed 6 Nov 2012]. French. Available from: http://.nrbc-sudest.org/public/public manuscrite. aspx?PAGE CODE=PUBLIC CONTEXTE COORD

3. Gilchrist MJ. A national laboratory network for bioterrorism: evolution from a prototype network of laboratories performing routine surveillance. Mil Med. 2000;165(7 Supple 2):28-31.

4. Peterson LR, Hamilton JD, Baron EJ, Tompkins LS, Miller JM, Wilfert CM, et al. Role of clinical microbiology laboratories in the management and control of infectious diseases and the delivery of health care. Clin Infect Dis. 2001;32(4):605-11.

5. Pien BC, Saah JR, Miller SE, Woods CW. Use of sentinel laboratories by clinicians to evaluate potential bioterrorism and emerging infections. Clin Infect Dis. 2006.42(9):1311-24.

6. Kalish BT, Gaydos, CA, Hsieh YH, Christensen BE, Carroll KC, Cannons A, et al. National survey of laboratory response network sentinel laboratory preparedness. Disaster Med Public Health Prep. 2009;3(2 Suppl):S17-23.

7. Mock M, Fouet A. Anthrax. Annu Rev Microbiol. 2001;55:647-71.

8. Loïez C, Herwegh S, Wallet F, Armand S, Guinet F, Courcol RJ. Detection of Yersinia pestis in sputum by real time PCR. J Clin Microbiol. 2003;41(10):4873-5.

9. Blondiaux N, Gaillot O, Courcol RJ. MALDI-TOF mass spectrometry to identify clinical bacterial isolates: evaluation in a teaching hospital in Lille. Pathol Biol. 2010;58(1):55-7.

10. Wagar EA, Mitchell MJ, Carroll KC, Beavis KG, Petti $C A$, Schlaberg R, et al. A review of sentinel laboratory performance: identification and notification of bioterrorism agents. Arch Pathol Med. 2010;134(10):1490-503.

11. Arrêté du 30 Avril 2012 fixant la liste des micro-organismes et toxines prévue à l'article L.5139-1 du code de la santé publique. [Decree of 30 April 2012 establishing the list of micro-organisms and toxins in Article L. 5139-1 Code of Public Health]. Official Journal No. 0109 from 10 May 2012; p. 8788. Text No. 111. French. Available from: http://www.legifrance. gouv.fr/affichTexte.do?cidTexte=JORFTEXTo00025837146\&date Texte $=$ \& categorieLien $=$ id

12. Merens A, Vaissaire J, Cavallo J-D, Le Doujet C, Gros C, Bigaillon $C$, et al. E-test for antibiotic susceptibility testing of Bacillus anthracis, Bacillus cereus and Bacillus thuringiensis: evaluation of a French collection. Intern J Antimicrob Agents. 2008;31(5):490-2.

13. Valade E, Vaissaire J, Merens A, Hernandez E, Gros C, Le Doujet $C$, et al. Susceptibility of 71 French isolates of Francisella tularensis subsp. holarctica to eight antibiotics and accuracy of E-test $₫$ method. J Antimicrob Chemother. 2008;62(1):208-10. 